УДК 532.51

\title{
Unsteady 2D Motions a Viscous Fluid Described by Partially Invariant Solutions to the Navier-Stokes Equations
}

\author{
Victor K. Andreev* \\ Institute of Computational Modelling RAS SB \\ Akademgorodok, 50/44, Krasnoyarsk, 660036 \\ Institute of Mathematics and Computer Science \\ Siberian Federal University \\ Svobodny, 79, Krasnoyarsk, 660041
}

Russia

Received 10.02.2015, received in revised form 03.03.2015, accepted 30.03.2015

$\overline{3 D}$ continuous subalgebra is used to searching partially invariant solution of viscous incompressible fluid equations. It can be interpreted as a $2 D$ motion of one or two immiscible fluids in plane channel. The arising initial boundary value problem for factor-system is an inverse one. Unsteady problem for creeping motions is solved by separating of variables method for one fluid or Laplace transformation method for two fluids.

Keywords: partially invariant solution, viscous fluid, free boundary problem, interface.

\section{Introduction}

The Navier-Stokes equations for 2D motions of a viscous fluid are recorded by

$$
\begin{aligned}
& u_{t}+u u_{x}+v u_{y}+\frac{1}{\rho} p_{x}=\nu\left(u_{x x}+u_{y y}\right), \\
& v_{t}+u v_{x}+v v_{y}+\frac{1}{\rho} p_{y}=\nu\left(v_{x x}+v_{y y}\right)-g, \\
& u_{x}+v_{y}=0,
\end{aligned}
$$

where $\rho$ is the constant fluid density, $u$ and $v$ are the velocity components in the $x$ and $y$ directions, respectively, $p$ is the pressure and $g$ is the gravity acceleration, $\nu$ is the fluid viscosity. The group of point transformations admitted by the system $(0.1)$ is computed in $[1,2]$. Corresponding this group basic continuous Lie algebra includes the three parametrical subalgebra $\left\langle\partial_{x}, \partial_{u}+t \partial_{x}, \partial_{p}\right\rangle$. It has the invariants $t, y, v$ and partly invariant solution of (0.1) rang two and defect two necessary to seek in the form $u=u(x, y, t), v=v(y, t), p=p(x, y, t)$. From continuity equation $u_{x}+v_{y}=0$ we obtain the relations

$$
u(x, y, t)=w(y, t) x+u_{1}(y, t), \quad w(y, t)+v_{y}(y, t)=0 .
$$

Navier-Stokes equations (0.1) are equivalent to the system

$$
\begin{aligned}
& w_{t}+v w_{y}+w^{2}=f(t)+\nu w_{y y}, \quad \frac{1}{\rho} p=l(y, t)-f(t) \frac{x^{2}}{2}-g y, \\
& v=-\int_{0}^{y} w(z, t) d z, \quad l_{y}=\nu v_{y y}-v_{t}-v v_{y} \\
& u_{1 t}+v u_{1 y}+w u_{1}=\nu u_{1 y y} .
\end{aligned}
$$

In what follows we assume that $u_{1}(y, t)=0$.

*andr@icm.krasn.ru

(c) Siberian Federal University. All rights reserved 


\section{Flow in layer with two rigid walls}

In this section the solution (0.2) under consideration shall be interpreted as $2 \mathrm{D}$ motion viscous liquid fills the layer $0<y<h$ with a rigid walls $y=0, y=h=$ const. Let us attach the initial and boundary conditions

$$
\begin{gathered}
w(y, 0)=w_{0}(y), \quad w_{0}(0)=w_{0}(h)=0, \quad \int_{0}^{h} w_{0}(z) d z=0 \\
w(0, t)=w(h, t)=0, \quad \int_{0}^{h} w(z, t) d z=0 .
\end{gathered}
$$

Thus, the function $w(y, t)$ is the solution of integro-differential equation

$$
w_{t}-w_{y} \int_{0}^{y} w(z, t) d z+w^{2}=\nu w_{y y}+f(t)
$$

with initial and boundary conditions (1.1), (1.2).

Here and further suppose the Reynolds number $\operatorname{Re}=\max _{y \in[0,1]}\left|w_{0}(y)\right| h^{2} / \nu \ll 1$. In such case we can neglect the nonlinear terms in equation (1.3) and the following initial boundary value problem is arised

$$
\begin{gathered}
w_{t}=\nu w_{y y}+f(t), \quad y \in(0, h), \quad t>0 ; \\
w(y, 0)=w_{0}(y) \\
w(0, t)=0, \quad w(h, t)=0, \quad \int_{0}^{h} w(y, t) d y=0 .
\end{gathered}
$$

Integrating equation (1.4) we obtain function $f(t)$

$$
f(t)=\frac{\nu}{h}\left(w_{y}(0, t)-w_{y}(h, t)\right), \quad f(0)=\frac{\nu}{h}\left(w_{0 y}(0)-w_{0 y}(h)\right) .
$$

Hence, we deduce the so-called loaded equation

$$
w_{t}=\nu w_{y y}+w_{y}(1, t)-w_{y}(0, t) .
$$

But we determine new function $W(y, t)=w_{y}(y, t)$. It satisfies the problem

$$
\begin{gathered}
W_{t}=\nu W_{y y}, \quad y \in(0,1), \quad t>0 \\
W(y, 0)=w_{0 y} \\
\int_{0}^{h} y W(y, t) d y=0 \\
\int_{0}^{h} W(y, t) d y=0
\end{gathered}
$$

This problem is not classical one for the heat equation (1.4).

The problem (1.8)-(1.11) has the exact solution

$$
W(y, t)=\sum_{k=1}^{\infty} a_{k} \exp \left(-\frac{4 \lambda_{k}^{2} \nu t}{h^{2}}\right) \sin \left[\frac{\lambda_{k}(2 y-h)}{h}\right] .
$$

where $\lambda_{k}$ is $k$ th positive root of the equation

$$
\begin{aligned}
\operatorname{tg} \lambda_{k}=\lambda_{k}, \quad \lambda_{k} & \rightarrow(2 k+1) \pi / 2, \quad k \rightarrow \infty . \\
& -141-
\end{aligned}
$$


A constants $a_{k}$ are the Fourier series coefficients of known function $w_{0 y}$, i.e.

$$
w_{0 y}=\sum_{k=1}^{\infty} a_{k} \sin \left[\frac{\lambda_{k}(2 y-h)}{h}\right]
$$

Hence, from (1.7)

$$
\begin{aligned}
f(t)=\frac{\nu}{h}[W(0, t)-W(h, t)]=-\frac{2 \nu}{h} \sum_{k=1}^{\infty} a_{k} \exp \left(-\frac{4 \lambda_{k}^{2} \nu t}{h^{2}}\right) \sin \lambda_{k}= & \\
& =-\frac{2 \nu}{h} \sum_{k=1}^{\infty} a_{k} \frac{\lambda_{k}}{\sqrt{1+\lambda_{k}^{2}}} \exp \left(-\frac{4 \lambda_{k}^{2} \nu t}{h^{2}}\right) .
\end{aligned}
$$

Functions $w(y, t)$ and velocity component $v(y, t)$ can be found by the formulae

$$
\begin{gathered}
w(y, t)=\frac{h}{2} \sum_{k=1}^{\infty} \frac{a_{k}}{\lambda_{k}} \exp \left(-\frac{4 \lambda_{k}^{2} \nu t}{h^{2}}\right)\left\{\cos \lambda_{k}-\cos \left[\frac{\lambda_{k}(2 y-h)}{h}\right]\right\}, \\
v(y, t)=\frac{h}{2} \sum_{k=1}^{\infty} \frac{a_{k}}{\lambda_{k}} \exp \left(-\frac{4 \lambda_{k}^{2} \nu t}{h^{2}}\right)\left\{\frac{h}{2 \lambda_{k}}\left[\sin \left(\frac{\lambda_{k}(2 y-h)}{h}\right)+\sin \lambda_{k}\right]-y \cos \lambda_{k}\right\} .
\end{gathered}
$$

\section{Flow in layer with one rigid wall and free boundary}

In the same assumptions like section 1 the function $w(y, t)$ is governed by the equation (1.4). The initial data and boundary conditions are (1.5), (1.6), but it is necessary to change second condition in $(1.6)$ on $w_{y}(h, t)=0$. For the function $f(t)$ one obtains

$$
f(t)=\frac{\nu}{h} w_{y}(0, t)
$$

As concerning function $W(y, t)=w_{y}(y, t)$ it satisfies the equation (1.8) with initial data (1.9) and boundary conditions

$$
W(h, t)=0, \quad \int_{0}^{h}(h-y) W(y, t) d y=0 .
$$

Using separation of variables technique the problem can be solved to obtain $W$ :

$$
W(y, t)=\frac{2}{h} \sum_{k=1}^{\infty} b_{k} \frac{\left(1+\lambda_{k}^{2}\right)}{\lambda_{k}^{2}} \exp \left(-\frac{\lambda_{k}^{2} \nu t}{h^{2}}\right) \sin \left[\frac{\lambda_{k}(h-y)}{h}\right]
$$

Therefore

$$
w(y, t)=2 \sum_{k=1}^{\infty} b_{k} \frac{\left(1+\lambda_{k}^{2}\right)}{\lambda_{k}^{3}} \exp \left(-\frac{\lambda_{k}^{2} \nu t}{h^{2}}\right)\left\{\cos \left[\frac{\lambda_{k}(h-y)}{h}\right]-\cos \lambda_{k}\right\}
$$

and from (2.1), (0.2) we get

$$
f(t)=\frac{2 \nu}{h^{2}} \sum_{k=1}^{\infty} b_{k} \frac{\sqrt{1+\lambda_{k}^{2}}}{\lambda_{k}} \exp \left(-\frac{\lambda_{k}^{2} \nu t}{h^{2}}\right)
$$




$$
v(y, t)=2 \sum_{k=1}^{\infty} b_{k} \frac{\left(1+\lambda_{k}^{2}\right)}{\lambda_{k}^{3}} \exp \left(-\frac{\lambda_{k}^{2} \nu t}{h^{2}}\right)\left\{y \cos \lambda_{k}+\frac{h}{\lambda_{k}}\left[\sin \left(\frac{\lambda_{k}(y-h)}{h}\right)+\sin \lambda_{k}\right]\right\} .
$$

A constants $b_{k}$ are the Fourier series coefficients of function $w_{0 y}$, i.e.

$$
w_{0 y}=\frac{2}{h} \sum_{k=1}^{\infty} b_{k} \frac{\left(1+\lambda_{k}^{2}\right)}{\lambda_{k}^{2}} \sin \left[\frac{\lambda_{k}(h-y)}{\lambda_{k}}\right] .
$$

\section{Layered motion of two immiscible fluids}

Let us consider a system of two immiscible fluids separated by the interface $y=h_{1}$. The parameters of the fluid moving in the band $0<y<h_{1}, x \in R$ are indicated by the subscript "1", and the parameters of the fluid moving in the band $h_{1}<y<h, x \in R$ are indicated by the subscript " 2 ". In the plane motion considered here, the functions $w_{j}(y, t)$ and $f_{j}(t), j=1,2$, are the solutions of the equations

$$
w_{j t}=\nu_{j} w_{j y y}+f_{j}(t)
$$

related by the conditions on the interface [3]

$$
\begin{gathered}
\int_{0}^{h_{1}} w_{1}(z, t) d z=0 ; \\
w_{1}\left(h_{1}, t\right)=w_{2}\left(h_{1}, t\right), \quad \mu_{1} w_{1 y}\left(h_{1}, t\right)-\mu_{2} w_{2 y}\left(h_{1}, t\right)=0 ;
\end{gathered}
$$

the no-slip conditions on the solid boundaries of the flow domain

$$
\begin{gathered}
w_{1}(0, t)=0, \quad w_{2}(h, t)=0 ; \\
\int_{h_{1}}^{h} w_{2}(z, t) d z=0,
\end{gathered}
$$

and initial data

$$
\begin{array}{ll}
w_{1}(y, 0)=w_{10}(y), & 0 \leqslant y \leqslant h_{1}, \\
w_{2}(y, 0)=w_{20}(y), & h_{1} \leqslant y \leqslant h .
\end{array}
$$

Remark 1. The initial value problem (3.1)-(3.5) has not a solution expanded into a Fourier series.

A priori estimates. Using equalities (3.2), (3.4) and integrating equation (3.1) we obtain the relations

$$
\begin{aligned}
& f_{1}(t)=\frac{\nu_{1}}{h_{1}}\left[w_{1 y}(0, t)-w_{1 y}\left(h_{1}, t\right)\right], \quad f_{1}(0)=\frac{\nu_{1}}{h_{1}}\left[w_{10 y}(0)-w_{10 y}\left(h_{1}\right)\right], \\
& f_{2}(t)=\frac{\nu_{2}}{h-h_{1}}\left[w_{2 y}\left(h_{1}, t\right)-w_{2 y}(h, t)\right], \quad f_{2}(0)=\frac{\nu_{2}}{h-h_{1}}\left[w_{20 y}\left(h_{1}\right)-w_{20 y}(h)\right] .
\end{aligned}
$$

There exists the energetic identity for the problem (3.1)-(3.5)

$$
\frac{\partial}{\partial t} E(t)+\mu_{1} \int_{0}^{h_{1}} w_{1 y}^{2} d y+\mu_{2} \int_{h_{1}}^{h} w_{2 y}^{2} d y=0
$$

where

$$
E(t)=\frac{1}{2} \rho_{1} \int_{0}^{h_{1}} w_{1}^{2} d y+\frac{1}{2} \rho_{2} \int_{h_{1}}^{h} w_{2}^{2} d y .
$$


Lemma 1. The following inequality holds

$$
\int_{0}^{h_{1}} w_{1}^{2} d y+\int_{h_{1}}^{h} w_{2}^{2} d y \leqslant M\left(\mu_{1} \int_{0}^{h_{1}} w_{1 y}^{2} d y+\mu_{2} \int_{h_{1}}^{h} w_{2 y}^{2} d y\right)
$$

where $M$ is the solution of the variational problem

$$
M=\sup _{v_{1}, v_{2} \in V}\left[\frac{\int_{0}^{h_{1}} v_{1}^{2} d y+\int_{h_{1}}^{h} v_{2}^{2} d y}{\mu_{1} \int_{0}^{h_{1}} v_{1 y}^{2} d y+\mu_{2} \int_{h_{1}}^{h} v_{2 y}^{2} d y}\right] .
$$

Here $V \subset W_{2}^{1}\left(0, h_{1}\right) \times W_{2}^{1}\left(h_{1}, h\right)$ and conditions (3.3), (3.4) for $v_{1}, v_{2}$ are satisfied.

The proof is given in [4]. Due to this lemma we get $M=\left(h-h_{1}\right)^{2} / \mu_{1} z_{0}^{2}$, where $z_{0}$ is the minimal positive root of the equation

$$
\sin \left(a_{1} z\right) \cos \left(a_{2} z\right)+a_{2} \sin \left(a_{2} z\right) \cos \left(a_{1} z\right)=0 .
$$

Here $a_{1}=h_{1} /\left(h-h_{1}\right), a_{2}=\left(\mu_{1} / \mu_{2}\right)^{1 / 2}$. From (3.8), (3.9) we get inequality

$$
\frac{\partial E}{\partial t}+2 \delta E \leqslant 0, \quad \delta=\frac{1}{M} \min \left(\frac{1}{\rho_{1}}, \frac{1}{\rho_{2}}\right)
$$

hence

$$
E(t) \leqslant E(0) e^{-2 \delta t}
$$

with $E(0)=\frac{1}{2} \rho_{1} \int_{0}^{h_{1}} w_{10}^{2}(y) d y+\frac{1}{2} \rho_{2} \int_{h_{1}}^{h} w_{20}^{2}(y) d y$.

Moreovere, there is another identity for the problem (3.1)-(3.6)

$$
\rho_{1} \int_{0}^{h_{1}} w_{1 t}^{2} d y+\rho_{2} \int_{h_{1}}^{h} w_{2 t}^{2} d y+\frac{1}{2} \frac{\partial}{\partial t}\left(\mu_{1} \int_{0}^{h_{1}} w_{1 y}^{2} d y+\mu_{2} \int_{h_{1}}^{h} w_{2 y}^{2} d y\right)=0
$$

and then following estimates hold

$$
\int_{0}^{h_{1}} w_{1 y}^{2} d y \leqslant \frac{W_{0}}{\mu_{1}}, \quad \int_{h_{1}}^{h} w_{2 y}^{2} d y \leqslant \frac{W_{0}}{\mu_{2}}
$$

where

$$
W_{0}=\mu_{1} \int_{0}^{h_{1}} w_{10}^{2} d y+\mu_{2} \int_{h_{1}}^{h} w_{20}^{2} d y .
$$

From (3.4), (3.10), (3.11) we have the estimates

$$
\left|w_{j}(y, t)\right| \leqslant\left(\frac{8 E(0) W_{0}}{\nu_{j}}\right)^{1 / 4} e^{-\delta t / 2} .
$$

Therefore, the motion of fluids are slowed down by the viscous friction according to inequalities (3.12).

Now, let us go over to estimate the function $f_{j}(t)$ defined by $(3.7)$. Firstly, the new unknowns $V_{j}(y, t)=w_{j t}(y, t)$ are satisfies the problem (3.1)-(3.6) with $f_{j t}$ instead of $f_{j}(t)$ and initial data at $t=0$ equal to

$$
\begin{gathered}
V_{10}(y)=\nu_{1} w_{10 y y}(y)+\frac{\nu_{1}}{h_{1}}\left[w_{10 y}(0)-w_{10 y}\left(h_{1}\right)\right] \\
V_{20}(y)=\nu_{2} w_{20 y y}(y)+\frac{\nu_{2}}{h-h_{1}}\left[w_{20 y}\left(h_{1}\right)-w_{20 y}(h)\right] . \\
-144-
\end{gathered}
$$


Hence, we get estimates like (3.12)

$$
\left|w_{j t}(y, t)\right| \leqslant\left(\frac{8 E^{1}(0) W_{0}^{1}}{\nu_{j}}\right)^{1 / 4} e^{-\delta t / 2},
$$

here

$$
\begin{aligned}
E^{1}(0) & =\frac{1}{2} \rho_{1} \int_{0}^{h_{1}} V_{10}^{2}(y) d y+\frac{1}{2} \rho_{2} \int_{h_{1}}^{h} V_{20}^{2}(y) d y \\
W_{0}^{1} & =\mu_{1} \int_{0}^{h_{1}} V_{10 y}^{2}(y) d y+\mu_{2} \int_{h_{1}}^{h} V_{20 y}^{2}(y) d y .
\end{aligned}
$$

If we multiply equation $(3.1)$ by $y\left(h_{1}-y\right)(j=1)$ or $\left(y-h_{1}\right)(h-y)(j=2)$ and integrate, then we obtain equalities

$$
\begin{aligned}
\frac{h_{1}^{3}}{6} f_{1}(t) & =\int_{0}^{h_{1}} y\left(h_{1}-y\right) w_{1 t}(y, t) d y-\nu_{1} h_{1} w_{1}\left(h_{1}, t\right), \\
\frac{\left(h-h_{1}\right)^{3}}{6} f_{2}(t) & =\int_{h_{1}}^{h}\left(y-h_{1}\right)(h-y) w_{2 t}(y, t) d y-\nu_{2}\left(h-h_{1}\right) w_{2}(h, t) .
\end{aligned}
$$

Using inequalities (3.12), (3.13) we get estimates

$$
\left|f_{j}(t)\right| \leqslant C_{j} e^{-\delta t / 2}
$$

with constants are

$$
\begin{gathered}
C_{1}=6\left(\frac{8 E^{1}(0) W_{0}^{1}}{\nu_{1}}\right)^{1 / 4}+\frac{6 \nu_{1}}{h_{1}^{2}}\left(\frac{8 E(0) W_{0}}{\nu_{1}}\right)^{1 / 4} \\
C_{2}=6\left(\frac{8 E^{1}(0) W_{0}^{1}}{\nu_{2}}\right)^{1 / 4}+\frac{6 \nu_{2}}{\left(h-h_{1}\right)^{2}}\left(\frac{8 E(0) W_{0}}{\nu_{2}}\right)^{1 / 4} .
\end{gathered}
$$

\section{Solution in the Laplace representation}

Let us apply the Laplace transform to problem (3.1)-(3.5)

$$
\tilde{w}_{j}(y, p)=\int_{0}^{\infty} w_{j}(y, t) e^{-p t} d t .
$$

As a result, we obtain a boundary-value problem for the ODE:

$$
\begin{gathered}
\tilde{w}_{j y y}-\frac{p}{\nu_{j}} \tilde{w}_{j}=-\frac{\tilde{f}_{j}(p)}{\nu_{j}}-\frac{w_{j 0}(y)}{\nu_{j}}, \quad j=1,2 ; \\
\tilde{w}_{1}(0, p)=0, \quad \tilde{w}_{2}(h, p)=0 ; \\
\tilde{w}_{1}\left(h_{1}, p\right)=\tilde{w}_{2}\left(h_{1}, p\right), \quad \mu_{1} \tilde{w}_{1 y}\left(h_{1}, p\right)-\mu_{2} \tilde{w}_{2 y}\left(h_{1}, p\right)=0 ; \\
\int_{0}^{h_{1}} \tilde{w}_{1}(y, p) d=0, \quad \int_{h_{1}}^{h} \tilde{w}_{2}(y, p) d y=0
\end{gathered}
$$

with the exact solution

$$
\begin{aligned}
\tilde{w}_{j}(y, p)=C_{j}^{1} \operatorname{sh}\left(\sqrt{\frac{p}{\nu_{j}}} y\right)+C_{j}^{2} \operatorname{ch}\left(\sqrt{\frac{p}{\nu_{j}}} y\right)- & \\
& -\frac{1}{\sqrt{p \nu_{j}}} \int_{y_{j}}^{y} w_{j 0}(z) \operatorname{sh}\left(\sqrt{\frac{p}{\nu_{j}}}(y-z)\right) d z+\frac{\tilde{f}_{j}(p)}{p}, \\
& -145-
\end{aligned}
$$


where $y_{1}=0, y_{2}=h$,

$$
\begin{gathered}
C_{1}^{1}=\left[\operatorname{ch}\left(\sqrt{\frac{p}{\nu_{1}}} h_{1}\right)-1\right]^{-1}\left\{\frac{\tilde{f}_{1}(p)}{p}\left[\operatorname{sh}\left(\sqrt{\frac{p}{\nu_{1}}} h_{1}\right)-\sqrt{\frac{p}{\nu_{1}}} h_{1}\right]+\right. \\
\left.\quad+\frac{1}{\nu_{1}} \int_{0}^{h_{1}}\left[\int_{0}^{y} w_{10}(z) \operatorname{sh}\left(\sqrt{\frac{p}{\nu_{1}}}(y-z)\right) d z\right] d y\right\} \\
C_{2}^{1}=\frac{1}{\Delta}\left\{G \operatorname{ch}\left(\sqrt{\frac{p}{\nu_{2}}} h\right)+\frac{\tilde{f}_{2}(p)}{p}\left[\operatorname{sh}\left(\sqrt{\frac{p}{\nu_{2}}} h\right)-\operatorname{sh}\left(\sqrt{\frac{p}{\nu_{2}}} h_{1}\right)\right]\right\} \\
C_{2}^{2}=\frac{1}{\Delta}\left\{\frac{\tilde{f}_{2}(p)}{p}\left[\operatorname{ch}\left(\sqrt{\frac{p}{\nu_{2}}} h_{1}\right)-\operatorname{ch}\left(\sqrt{\frac{p}{\nu_{2}}} h\right)\right]-G \operatorname{sh}\left(\sqrt{\frac{p}{\nu_{2}}} h\right)\right\} \\
C_{1}^{2}=-\frac{\tilde{f}_{1}(p)}{p}, \quad \Delta=1-\operatorname{ch}\left(\sqrt{\frac{p}{\nu_{2}}}\left(h-h_{1}\right)\right) \\
G=\frac{\left(h_{1}-h\right)}{\sqrt{p \nu_{2}}} \tilde{f}_{2}(p)+\frac{1}{\nu_{2}} \int_{h_{1}}^{h}\left[\int_{h}^{y} w_{20}(z) \operatorname{sh}\left(\sqrt{\frac{p}{\nu_{2}}}(y-z)\right) d z\right] d y
\end{gathered}
$$

Taking into account formulae (4.6), (4.7) from (4.5), we get

$$
\tilde{f}_{1}=\frac{p}{a_{1} a_{4}-a_{2} a_{3}}\left(G_{1} a_{4}-G_{2} a_{2}\right), \quad \tilde{f}_{2}=\frac{p}{a_{1} a_{4}-a_{2} a_{3}}\left(G_{2} a_{1}-G_{1} a_{3}\right)
$$

here

$$
\begin{gathered}
a_{1}=\left[\operatorname{ch}\left(\sqrt{\frac{p}{\nu_{1}}} h_{1}\right)-1\right]^{-1}\left[-2+2 \operatorname{ch}\left(\sqrt{\frac{p}{\nu_{1}}} h_{1}\right)-\sqrt{\frac{p}{\nu_{1}}} h_{1} \operatorname{sh}\left(\sqrt{\frac{p}{\nu_{1}}} h_{1}\right)\right] \\
a_{2}=-\frac{1}{\Delta}\left[2+\sqrt{\frac{p}{\nu_{2}}}\left(h-h_{1}\right) \operatorname{sh}\left(\sqrt{\frac{p}{\nu_{2}}}\left(h-h_{1}\right)\right)\right], \\
a_{3}=\mu_{1} \sqrt{\nu_{2}}\left\{\sqrt{\nu_{1}}\left[\operatorname{ch}\left(\sqrt{\frac{p}{\nu_{1}}} h_{1}\right)-1\right]\right\}^{-1}\left[\operatorname{sh}\left(\sqrt{\frac{p}{\nu_{1}}} h_{1}\right)-\sqrt{\frac{p}{\nu_{1}}} h_{1} \operatorname{ch}\left(\sqrt{\frac{p}{\nu_{1}}} h_{1}\right)\right] \\
a_{4}=\frac{\mu_{2}}{\Delta}\left[\sqrt{\frac{p}{\nu_{2}}}\left(h-h_{1}\right) \operatorname{ch}\left(\sqrt{\frac{p}{\nu_{2}}}\left(h-h_{1}\right)\right)-\operatorname{sh}\left(\sqrt{\frac{p}{\nu_{2}}}\left(h-h_{1}\right)\right)\right] \\
G_{1}=\frac{1}{\sqrt{p \nu_{1}}} \int_{0}^{h_{1}} w_{10}(z) \operatorname{sh}\left(\sqrt{\frac{p}{\nu_{1}}}\left(h_{1}-z\right)\right) d z+\frac{1}{\sqrt{p \nu_{2}}} \int_{h_{1}}^{h} w_{20}(z) \operatorname{sh}\left(\sqrt{\frac{p}{\nu_{2}}}\left(h_{1}-z\right)\right) d z- \\
-\frac{1}{\nu_{1}} \operatorname{sh}\left(\sqrt{\frac{p}{\nu_{1}}} h_{1}\right)\left[\operatorname{ch}\left(\sqrt{\frac{p}{\nu_{1}}} h_{1}\right)-1\right]^{-1} \int_{0}^{h_{1}}\left[\int_{0}^{y} w_{10}(z) \operatorname{sh}\left(\sqrt{\frac{p}{\nu_{1}}}(y-z)\right) d z\right] d y- \\
-\frac{1}{\nu_{2} \Delta} \operatorname{sh}\left(\sqrt{\frac{p}{\nu_{2}}}\left(h-h_{1}\right)\right) \int_{h_{1}}^{h}\left[\int_{h}^{y} w_{20}(z) \operatorname{sh}\left(\sqrt{\frac{p}{\nu_{2}}}(y-z)\right) d z\right] d y, \\
G_{2}=\frac{\mu_{1} \sqrt{\nu_{2}}}{\nu_{1} \sqrt{p}} \int_{0}^{h_{1}} w_{10}(z) \operatorname{ch}\left(\sqrt{\frac{p}{\nu_{1}}}\left(h_{1}-z\right)\right) d z+\frac{\mu_{2}}{\sqrt{p \nu_{2}}} \int_{h_{1}}^{h} w_{20}(z) \operatorname{ch}\left(\sqrt{\frac{p}{\nu_{2}}}\left(h_{1}-z\right)\right) d z- \\
-\frac{\mu_{1} \sqrt{\nu_{2}}}{\nu_{1}^{3 / 2}} \operatorname{ch}\left(\sqrt{\frac{p}{\nu_{2}}} h_{1}\right)\left[\operatorname{ch}\left(\sqrt{\frac{p}{\nu_{1}}} h_{1}\right)-1\right]_{0}^{-1} \int_{0}^{h_{1}}\left[\int_{0}^{y} w_{10}(z) \operatorname{sh}\left(\sqrt{\frac{p}{\nu_{1}}}(y-z)\right) d z\right] d y+ \\
+\frac{\mu_{2}}{\nu_{2} \Delta} \operatorname{ch}\left(\sqrt{\frac{p}{\nu_{2}}}\left(h-h_{1}\right)\right) \int_{h_{1}}^{h}\left[\int_{h}^{y} w_{20}(z) \operatorname{sh}\left(\sqrt{\frac{p}{\nu_{2}}}(y-z)\right) d z\right] d y .
\end{gathered}
$$


Simple, but cumbersome calculations with the use of asymptotic representations for functions $\operatorname{sh} x$ and $\operatorname{ch} x$ show that

$$
\lim _{t \rightarrow \infty} w_{j}(y, t)=\lim _{p \rightarrow 0} p \tilde{w}_{j}(y, p)=0, \quad \lim _{t \rightarrow \infty} f_{j}(t)=\lim _{p \rightarrow 0} p \tilde{f}(p)=0 .
$$

Lust results obtained are good agrement with the a priori estimates (3.13) and (3.14).

\section{Conclusions}

The partly invariant solution of Navier-Stokes equations is investigated. This solution may describes the plane unsteady motions of a viscous fluid in a strip with two rigid walls, the fluid motion with one rigid wall and free boundary or the motion of a two immiscible fluids with interface in a strip bounded rigid walls. The motion arised due to initial velocity field. It was shown that this problem can be reduced for creeping motions to the linear initial boundary inverse problem for parabolic equations. Two problem were solved by Fourier method. At that time, the interface problem is solved by using some properties of the Laplace transformation. For any cases the motions are retarded by viscous friction.

\section{References}

[1] V.O.Bytev, Group-theoretic properties of the Navier-Stokes equations, Chislennye metody sploshnoi mehaniki, 3(1972), no. 3, 13-17 (in Russian).

[2] V.K.Andreev, O.V.Kaptsov, V.V.Pukhnachev, A.A.Rodionov, Application of Group-Theoretical Methods in Hydrodynamics, Kluver Acad. Publ., Dordrecht, Boston, London, 2010.

[3] V.K.Andreev, Yu.A.Gaponenko, O.N.Goncharova, V.V.Pukhnachev, Mathematical Models of Convection, Walter de Gruyter GmbH and Co KG, Berlin/Boston, 2012.

[4] V.K.Andreev, On Inequalities of the Friedrichs type for Combined Domains, J. Siberian Federal Univ., Math. and Physics, 2(2009), no. 2, 146-157.

\section{Частично инвариантные решения уравнений Навье-Стокса, описывающие нестационарные двумерные движения вязкой жидкости}

Виктор К. Андреев

\footnotetext{
Трехмерная непрерывная подалгебра используется для нахождения частично инвариантного решения уравнений вязкой несжимаемой жидкости. Оно интерпретируется как двумерное движение одной или двух несмешивающихся жидкостей в плоском канале. Возникающая начальнокраевая задача для фактор-системы является обратной. Нестачионарная задача для ползущих движений решена методом разделения переменной для одной жидкости и методом преобразования Лапласа для двух жидкостей.
}

Ключевые слова: частично инвариантное решение, вязкая жидкость, задача со свободной границей, поверхность раздела. 